\title{
Partitioning and transmutation contribution of MYRRHA to an EU strategy for HLW management and main achievements of MYRRHA related FP7 and H2020 projects: MYRTE, MARISA, MAXSIMA, SEARCH, MAX, FREYA, ARCAS
}

\author{
Hamid Aït Abderrahim ${ }^{1,}$, Peter Baeten ${ }^{1}$, Alain Sneyers ${ }^{1}$, Marc Schyns ${ }^{1}$, Paul Schuurmans ${ }^{1}$, Anatoly Kochetkov ${ }^{1}$, \\ Gert Van den Eynde ${ }^{1}$, and Jean-Luc Biarrotte ${ }^{2}$ \\ ${ }^{1} \mathrm{SCK} \bullet \mathrm{CEN}$, Boeretang 200, $2400 \mathrm{Mol}$, Belgium \\ ${ }^{2}$ CNRS/IN2P3, 3 rue Michel-Ange, 75016 Paris, France
}

Received: 31 July 2019 / Accepted: 18 September 2019

\begin{abstract}
Today, nuclear power produces $11 \%$ of the world's electricity. Nuclear power plants produce virtually no greenhouse gases or air pollutants during their operation. Emissions over their entire life cycle are very low. Nuclear energy's potential is essential to achieving a deeply decarbonized energy future in many regions of the world as of today and for decades to come, the main value of nuclear energy lies in its potential contribution to decarbonizing the power sector. Nuclear energy's future role, however, is highly uncertain for several reasons: chiefly, escalating costs and, the persistence of historical challenges such as spent fuel and radioactive waste management. Advanced nuclear fuel recycling technologies can enable full use of natural energy resources while minimizing proliferation concerns as well as the volume and longevity of nuclear waste. Partitioning and Transmutation (P\&T) has been pointed out in numerous studies as the strategy that can relax constraints on geological disposal, e.g. by reducing the waste radiotoxicity and the footprint of the underground facility. Therefore, a special effort has been made to investigate the potential role of $\mathrm{P} \& \mathrm{~T}$ and the related options for waste management all along the fuel cycle. Transmutation based on critical or sub-critical fast spectrum transmuters should be evaluated in order to assess its technical and economic feasibility and capacity, which could ease deep geological disposal implementation.
\end{abstract}

\section{Introduction}

Utilization of nuclear energy from fission reaction of uranium $(\mathrm{U})$ and plutonium $(\mathrm{Pu})$ produces high level radioactive waste (HLW) including minor actinides and fission products. For example, the EU presently relies on nuclear energy for $\sim 30 \%$ of its electric power production from Generation II and III nuclear fission reactors leading to the annual production of $2500 \mathrm{t} / \mathrm{y}$ of used fuel, containing about $25 \mathrm{t}$ of plutonium, and about $100 \backslash \mathrm{t}$ of HLW containing $3.5 \mathrm{t}$ of MAs, namely, neptunium (Np), americium $(\mathrm{Am})$ and curium $(\mathrm{Cm})$, and $3 \mathrm{t}$ of long-lived fission products (LLFPs). These MA and LLFP stocks need to be managed in an appropriate way. The used fuel reprocessing followed by the geological disposal (closed fuel cycle) or the direct geological disposal (open fuel cycle) are today the envisaged solutions, depending on national fuel cycle options and waste management policies. The required

\footnotetext{
* e-mail: hamid.ait.abderrahim@sckcen.be
}

time scale for geological disposal exceeds our accumulated technological knowledge and this remains the main concern of the general public. Partitioning and Transmutation $(\mathrm{P} \& \mathrm{~T})$ has been pointed out in numerous studies [1-9] as the strategy that can relax constraints on geological disposal and reduce the monitoring period to technological and manageable time scales (few hundreds of years). Therefore, a special effort has been made to integrate P\&T in advanced fuel cycles and advanced options for HLW management. Transmutation based on critical or subcritical fast spectrum transmuters should be evaluated in order to assess the technical and economic feasibility of this waste management option, which could ease the development of a deep geological disposal.

\section{Status today}

In most cases and various countries in EU such as France, UK, Belgium, Germany, Spain, Sweden, Italy as well as Japan, USA, Russia, South Korea, R\&D and/or 
Table 1. P\&T building blocks.

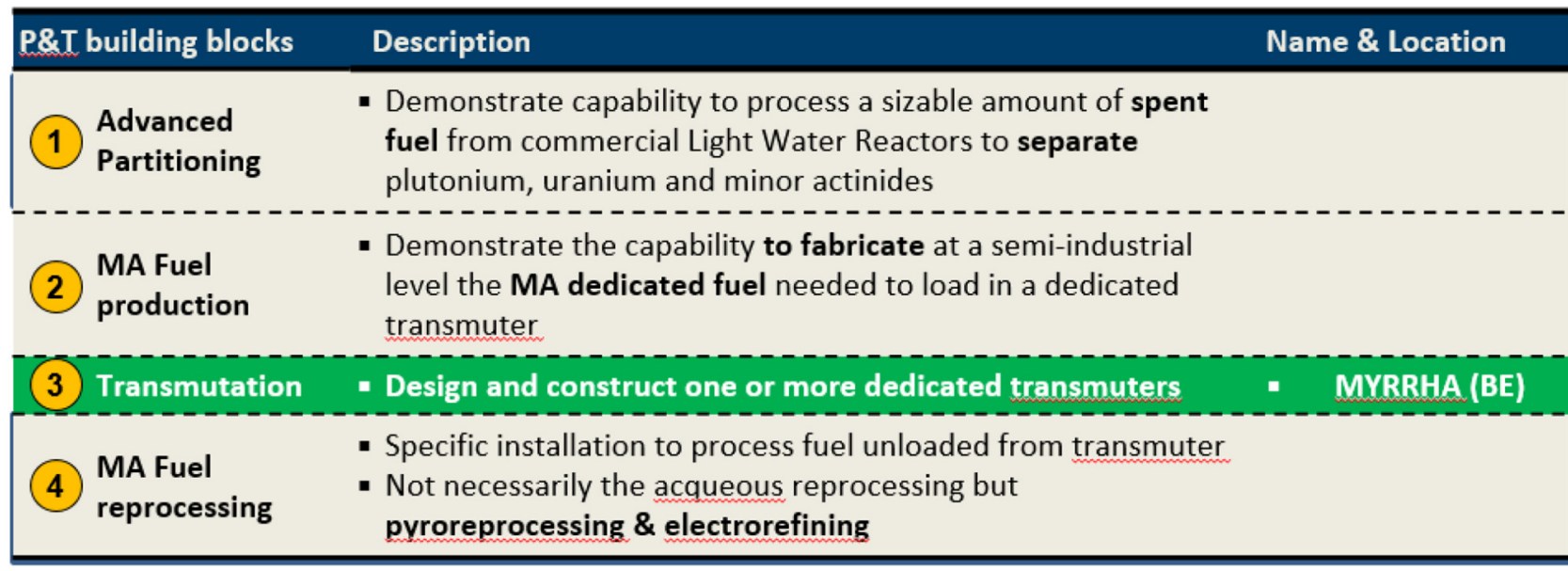

demonstration/validation/qualification programmes and infrastructures related to the advanced options for HLW management through P\&T and ADS development already exist for more than four decades IAEA-LMFNS [10] OECD/NEA DataBase for WPFC or Experimental Facilities [11,12]. In 2005, the research community on P\&T within the EU in collaboration with the DG Research \& Innovation of the European Commission started structuring its research towards a more integrated approach. This resulted in a European strategy based on the so-called four building blocks at engineering level for P\&T as summarized below:

- demonstration of the capability to process a sizable amount of spent fuel from commercial LWRs in order to separate plutonium $(\mathrm{Pu})$, uranium $(\mathrm{U})$ and minor actinides (MA) from $\mathrm{Pu}$ based spent fuels;

- demonstration of the capability to fabricate at a semiindustrial level the dedicated fuel sub-assembly to be loaded in a dedicated transmuter;

- design and construction of one or more dedicated transmuters;

- provision of a specific installation for processing of the dedicated fuel unloaded from the transmuter, which can be of a different type than the one used to process the original spent fuel unloaded from the commercial power plants, together with the fabrication of new dedicated fuel.

The four building blocks illustrated in Table 1 must be consistently developed in parallel. This approach is applicable in NI2050 [13] and will result in the identification of the costs and the benefits of P\&T for closing the fuel cycle and solving the SNF legacy.

The Belgian Government decision on September 7, 2018, to build in Mol the new large research infrastructure MYRRHA is a first sign of the realization of the building block 3 here above. Belgium allocated budget of $558 \mathrm{M} €$ for the period 2019-2038 that would allow building the phase one of MYRRHA consisting in a linear accelerator up to $100 \mathrm{MeV}$ coupled to a Proton Target facility (called MINERVA) and that will be operational around 2026. The same decision foresees the financing of the further development of the upgrade of the linac towards 600 $\mathrm{MeV}$ (phase 2) and of the MYRRHA sub-critical reactor (phase 3 ) including the support $R \& D$ and licensing work.

\subsection{Advanced partitioning}

Recycling of plutonium in the nuclear fuel cycle has been established on an industrial scale, leading, for example, to the use of MOX fuel in power reactors. Once $\mathrm{Pu}$ has been removed, the main contributor to the radiotoxicity and heat load of the remaining material is americium. In the past decade, a number of options have been developed and improved to separate Am from the PUREX rafinate. The first process of this kind, called EXAM, was designed at CEA in the 2010s. It was based on the previous DIAMEXSANEX process that aimed at co-extraction of Am and Curium. The key development was the creation of the suited molecule on which the selective stripping of Am is based. The first test molecule TEDGA (tetraethyldiglycolamide) was in a second phase replaced by TPEAN. Although this molecule showed enhanced selectivity on a lab scale, spiked tests of the EURO-EXAM process were not sufficiently successful to elevate TPEAN as the new reference molecule.

\subsection{MA fuel production}

Minor actinide fuel production has been established on a lab scale where it has been shown that the production of targets and small full segments is feasible.

\subsection{Transmutation}

In the field of transmutation, a distinction needs to be made between the behaviour under irradiation of MA fuel, i.e. the study of the transmutation process itself on the one hand and the development of the transmuter itself on the other hand. Transmutation studies have been carried out in the 
past using fast sodium cooled critical reactors and dedicated positions in material test reactors. Both homogeneous transmutation, with MA diluted at a low content $(<5 \%)$ in the standard driver fuel $(\mathrm{U}, \mathrm{Pu}) \mathrm{O} 2$ or by heterogeneous recycling with MA concentrated (10-15\%) in UO2 based fuels into the radial blankets (outer part of the core) have been tested. As mentioned above, the transmutation tests have been performed in test reactors that were not designed for transmutation. Although in the development of the new GenIV fast spectrum critical reactors such as ALFRED and ASTRID, transmutation is envisaged, the track towards the development of a dedicated transmuter on an industrial scale runs via the MYRRHA project. A conceptual design for an ADS based transmuter, EFIT, has been developed in the FP6 IPEUROTRANS. EFIT is a $400 \mathrm{MWth}$ Accelerator Driven System driven by a LINAC delivering $800 \mathrm{MeV}$ protons at a maximal current of $20 \mathrm{~mA}$ (typical operation would require $13 \mathrm{~mA}$ ). The system is a pool-type reactor and the core is cooled by pure lead. The fuel types considered are Inert Matrix Fuels (IMFs) such as CERCER (CeramicCeramic) and CERMET (Ceramic-Metal).

\subsection{MA fuel processing}

As the final building block in the Partitioning \& Transmutation strategy, one has to consider the reprocessing of the dedicated transmutation fuel. The irradiation times required for an efficient transmutation are rather long and it is uncertain if a single cladding could withstand these harsh conditions. It might also be necessary to adjust the plutonium content of the fuel to compensate for the loss in reactivity (some plutonium will be burned). As with the reprocessing of LWR fuel, also here the technologies can be separated in two groups: the aqueous technologies and the pyro technologies. These innovative fuels pose heavy challenges for the aqueous processes as they will contain significant amounts of plutonium and minor actinides that will pose problems for the stability of the chemicals (both solvents and extractants) used in the processes. The technologically most simple option is just to increase (significantly) the cooling-down period. However, this will have strong negative consequences on the time-scales needed to perform the irradiations. Advanced PUREX and GANEX processes are under development, but only still at laboratory scale. Since pyroprocessing does not rely on solvents and extractants, it will suffer less from the high radiation output of these innovative fuels. However, all technologies from the pyro family are still only available at lab scale.

\section{How to improve/accelerate through cooperation}

Based on the works performed in the SACSESS and GENIORS projects, a new process concept (AMSEL), relying on promising new molecule families developed for the GANEX and i-SANEX processes was proposed. The next step in the development is the validation of this newly proposed flowsheet. The basic idea of the AMSEL process is to selectively strip Am from an organic solvent containing
Am, $\mathrm{Cm}$ and lanthanide fission products. For this purpose the behaviour of both Am and fission product behaviour needs to be investigated. For the latter it is important to investigate whether they will follow the Am in the process. In addition the radiolytic stability of the system needs to be studied. Besides the research aiming at collecting and generating basic data, effort needs to be put in the development of the process itself including proper modelling and flowsheeting and the experimental validation of the process.

The main objectives in the field of transmutation studies are focussed on the behaviour of $241 \mathrm{Am}$ in the transmutation process since it is the dominant contributor to the radiotoxicity of the nuclear waste after the removal of $\mathrm{Pu}$. An important step forward is to bring robustness, accuracy and predictability to Fuel Performance Codes (FPCs), which are the cornerstone of fuel behaviour evaluation and safety analyses. In the context of transmutation, the specific focus is on investigating Ambearing fuel safety-related behaviour. For this purpose, three steps need to be taken. Firstly, one needs to Extend the validation database of models and simulation codes through the generation of data related to the production and behaviour under irradiation of helium, fission gases and fission products and to the specific thermo-chemical properties of fuels containing Am. Secondly, we must improve the prediction capabilities of FPCs by developing and implementing more reliable mechanistic models, and by moving towards coupling of FPCs with neutronics/ thermal-hydraulics codes, for the simulation of normal, offnormal and accidental conditions. Finally, we have to identify the experimental needs for code validation in offnormal situations, leading to the pre-design of a simulation-based transient irradiation tests that can be performed in a dedicated transmuter. Here collaboration between the different groups active in transmutation studies in Europe should be encouraged.

In the development of the transmuter, MYRRHA has come to a stage where the licensing process is aimed to be completed by 2026 and the redaction of the required documents including the preliminary safety assessment report (PSAR) should be completed by 2024. As a result, research supporting this effort by delivering input for the PSAR is required to make significant steps forward. Particularly, efforts should be focussed on the safety of the driver fuel and the fuel assembly and core arrangement in off normal conditions including transients, fuel blockages and fuel assembly deformations. The primary system safety should be focussed on the coolability of the system under all circumstances including the investigation of heat transfer and natural circulation in a pool configuration. In addition, sufficient effort needs to be put in the assessment of radiological release from the system, in particular in accident scenarios.

\section{Contribution of MYRRHA to the EU strategy towards industrialization of $P \& T$}

It is clear that due to the sheer size and cost of an installation like EFIT, one should work on smaller 
prototypical systems, for all the four building blocks in the European strategy. Moreover, the ADOPT frame work [6] also indicated that a demonstrator facility operating at a power of 50-100 MWth should be constructed as a stepping stone towards EFIT. MYRRHA, as a small-scale Accelerator Driven System that can provide fast neutrons for irradiation purposes, is put forward by SCK•CEN and recognized by the European Commission as a likely demonstrator. MYRRHA as an ADS Demo has the important objectives ${ }^{1}$ to:

- demonstrate the Accelerator Driven System technology

- demonstrate the reliability of the proton accelerator;

- demonstrate the coupling of a proton accelerator and sub-critical core at sufficient power;

- demonstrate the heavy liquid metal technology;

- demonstrate the feasibility of transmutation in such a system by being able to load sample-sized and pin-sized innovative ADS fuel materials for transmutation research;

- provide representative irradiation conditions in support of

- material qualification programs for EFIT;

- innovative ADS fuel qualification programs for EFIT.

To design and construct MYRRHA, a series of R\&D programs have been launched in the field of accelerator technology, heavy-liquid metal technology and reactor physics (the coupling of an accelerator to a subcritical core). SCK $\bullet$ CEN has established HLM labs for corrosion, for thermal-hydraulic experiments, lead and lead-bismuth chemistry, for component testing etc. All this research and development are essential for MYRRHA but contribute on a larger scale to the design and development of the larger EFIT facility.

\section{FP7 and H2020 MYRRHA related projects and their main achievements}

Since the establishment of the four building blocks strategy the fostering of the R\&D programme within the DG RTD programme for $\mathrm{P} \& \mathrm{~T}$ and waste management via the closed fuel cycle, became more evident and led to booking very important results to the programme and the $R \& D$ community driving this research. In the next paragraphs of this chapter we are illustrating this progress by summarizing seven projects of FP7 and H2020 related to the subject as well as their main achievements.

\subsection{MYRTE (MYRRHA Research and Transmutation Endeavour)}

The goal of MYRTE is to perform the necessary research in order to demonstrate the feasibility of transmutation of high-level waste at industrial scale through the development of the MYRRHA research facility. Within MYRRHA as a large research facility, the demonstration of the technological performance of transmutation will be

\footnotetext{
${ }^{1}$ MYRRHA has other objectives (radioisotope production, for one) of course, but they are not of relevance for this report.
}

combined with the use for the production of radio-isotopes and as a material testing for nuclear fission and fusion applications. Numerical studies and experimental facilities are foreseen to reach this goal.

\subsection{H2020 MYRTE - main achievements}

The MYRRHA Linac has to deliver a high-power proton beam with very high reliability and with minimum beam losses. The emphasis within MYRTE is on the injector which is considered to be the most critical part. The proton source and the low energy beam transport section have been put into operation successfully. The construction of the first accelerating structure, the 4-Rod Radio Frequency Quadrupole (RFQ), has been completed and pre-conditioning has been performed successfully. To feed the RFQ a $192 \mathrm{~kW}$ continuous wave Radio Frequency (RF) amplifier has been developed. To control the RF phases and amplitudes of the injector cavities a Low-Level RF control system is required. The design of the digital system is finished, and the system is ready to be used for the RFQ high power RF and beam tests. The control system for the $\mathrm{RFQ}$ is ready for first tests. Several diagnostics devices have been designed and prototypes have been realized. A reliability model of LINAC-4 at CERN has been developed and is under validation with data from operation. Prototypes of the Drift Tube Linac-cavities have been performed successfully. As result, all cavities exceeded the MYRRHA specifications.

In the thermal hydraulics work package, experiments and simulations go hand in hand. The flow induced vibration experiments have been finished successfully. Two independent approaches implemented in different code platforms have been developed to simulate flow-induced vibrations and have already been applied to determine preliminary modal characteristics of a MYRRHA rod bundle.

Volatile radioactive nuclides will be formed in the coolant of the MYRRHA reactor. Therefore, it is important to study chemical reactions that govern the potential release of these nuclides from the coolant to the gaseous environment. The main outcome of previous projects was that volatile species of nuclides form in presence of moisture and when oxide layers are present on the liquid metal. Currently, evaporation experiments are performed to study systematically the influence of moisture and oxygen content in the gas and the oxygen concentration in the liquid metal. These experiments are supported by theoretical studies. Also, the deposition of volatile molecules on surfaces of different materials is studied, with the purpose of finding materials that can be used to remove them from the gas phase. Very encouraging results have been obtained so far. These studies are performed on the most important fission products.

Thanks to the sub-criticality of the reactor, the fuel composition is more flexible for ADS than for a critical reactor, allowing a larger amount of minor actinides in the fuel. However, these advantages hold as long as the reactor remains subcritical. Thus, online reactivity monitoring is essential. Several methods of sub-criticality determination including both planned to be applied for ADS and reference 


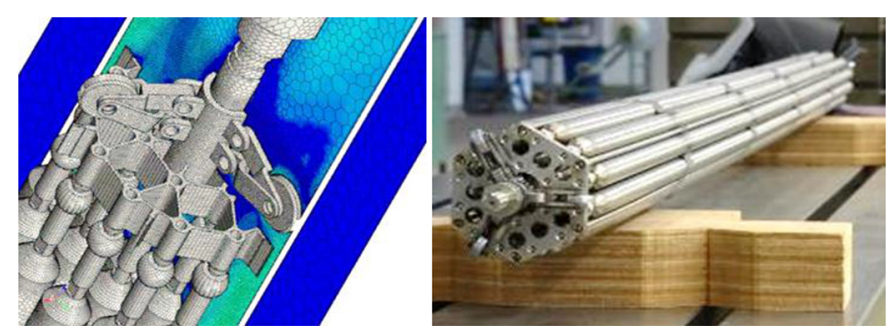

Fig. 1. MYRRHA control rod qualification.

ones were used and compared in MYRTE. The positions and deposit of the detectors used for the sub-criticality measurements are of high importance. This subject was thoroughly investigated. The experiments dedicated to the safety issues such as coolant and moderator voiding were completed. The calculations are in acceptable agreement with the experiments.

A specific work package in MYRTE is investigating topic issues related to the safe use of (U,Am)O2-x fuel as basis for transmutation of Am. Samples of sub-stoichiometric (U,Am)O2-x have been prepared, and their thermal diffusivity was measured in the temperature range between 500 and $1600 \mathrm{~K}$. Fuel to liquid lead bismuth metal interaction tests have been performed on representative (U,Am) $\mathrm{O} 2-\mathrm{x}$ samples in contact with $\mathrm{LBE}$ at $500{ }^{\circ} \mathrm{C}$ for $50 \mathrm{~h}$ under oxidizing and non-oxidizing conditions. The samples were characterized afterwards and no significant changes or interaction products were found.

\subsection{MARISA (MYRRHA Research Infrastructure Support Action)}

The FP7 project MARISA reviewed advanced fuel cycles and approaches for the long-term management of radioactive waste considered in the EU and nations worldwide. Work performed as part of MARISA confirmed the foremost role of MYRRHA in developing and demonstrating the concept of $\mathrm{P} \& \mathrm{~T}$ with the long-term objective of industrial deployment. Furthermore, research capabilities offered by MYRRHA will allow for integrating diverse national and international research programmes on Partitioning \& Transmutation.

\subsection{FP7 MARISA - main achievements}

The main achievements of MARISA have been the confirmation of positioning of MYRRHA as an International Open Users Facility in the European and global research landscape; MYRRHA legal structure, articles of association, intergovernmental agreements, governing rules, procedures for in-kind contributions and IPR defined; MYRRHA management principles developed, management instruments implemented and access framework for User Groups and Communities detailed; MYRRHA financing mechanisms and instruments defined; MYRRHA Environmental Impact Assessment Report development initiated; Technical integration MYRRHA primary system design, accelerator and Balance of Plant accomplished.

\subsection{MAXSIMA (Methodology, Analysis and Experiments for the safety in MYRRHA Assessment)}

The goal of MAXSIMA is to contribute to the "safety in MYRRHA" assessment.

\subsection{FP7 MAXSIMA - main achievements [14,15]}

Neutronic and shielding analysis as well as transient analyses using system codes in support of the MYRRHA safety studies has been carried out. The following main topics of the MYRRHA safety analysis have been studied in specific tasks of the MAXSIMA project:

- design of the MYRRHA core (and required shielding studies) using 3D methods;

- study of a complete list of accidental events and analysis of input data uncertainty propagation in the safetyrelevant output parameters;

- analysis of a number of severe accident scenarios potentially leading to core disruption.

Also, the safety aspects of the fuel assemblies and the control and safety rods of the reactor core have been analysed. In the fuel assembly, the cooling of a partially blocked fuel rod bundle was experimentally investigated. A second experiment was carried out to validate the correct movement of buoyancy driven control. Both experiments were numerical supported by CFD simulations. See Figure 1 for the control rod qualification.

To demonstrate the safety level of a steam generator in the primary pool, a large scale experiment has been designed, constructed and successfully carried out. The goal of the experiment was to characterize the Steam Generator Tube Rupture (SGTR) event in a configuration relevant for MYRRHA. In parallel numerical tools have been verified and validated to support the design phase as well as the safety assessment of such solutions. Post-test analysis was able to predict pressure and temperature time trends in agreement with experimental data, providing a contribution to code validation for water-LBE interaction scenario in a large pool facility.

The TRIGA Annular Core Pulsing Reactor (ACPR) at INR-Pitesti was used as a testing facility for transient test experiments. Fuel test segments (UO2, DIN 1.4970 cladded) were designed and fabricated by SCK $\bullet$ CEN and were transported to INR-Pitesti (Romania). The objective of the tests is to establish the failure threshold, expressed in deposited energy in the fuel, for fast transients. All transient test results of the UO2 tests were reported, design for MOX fuel fabrication and the MOX fuels fabrication test results were issued. It is intended to carry out transient test experiments in a follow-up project. See Figure 2.

An enhanced innovative passive safety system for Decay Heat Removal (DHR) of heavy liquid metal cooled reactors was developed. For such reactors the systems dedicated to heat removal should also guarantee that the primary coolant is not brought to the so-called freezing or solidification condition. Simulations have been carried out by computational tools (RELAP5 and TRACE) showing that the system is able to fulfil the expectations. 


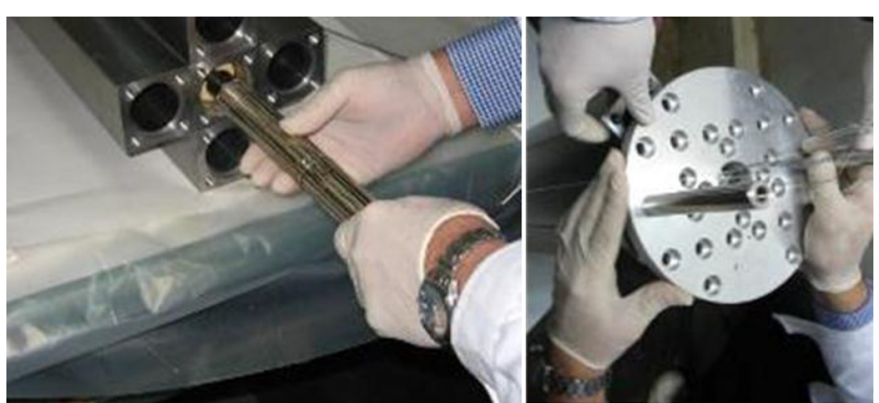

Fig. 2. MYRRHA fuel transient testing in TRIGA ACPR of ICN in Pitesti.

The records of the publications and dissemination activities can be summarised as follows: 10 journal papers, more than 80 contributions to national/international conferences/workshops and 20 contributions to lecture series/summer schools.

\subsection{SEARCH (Safe exploitation related chemistry for HLM reactors)}

The ESNII roadmap describes the deployment plan for advanced reactor systems in Europe. According to this plan, MYRRHA will be the first HLM cooled nuclear system to be realised. The SEARCH FP7 project was set up with the goal to support licensing MYRRHA by studying safety related aspects of the chemical behaviour of the liquid metal coolant and the fuel in the reactor. To achieve this goal different topics were be looked into. The first important objective was the examination of the methods to measure and control the oxygen content in the HLM coolant and to manage impurities in the melt which is essential in all conditions of reactor operation. A further step was taken by also investigating the chemical compatibility of the coolant with the nuclear fuel that will come into contact with each other in case of fuel pin leakage, melt or rupture. A complete analysis of these scenarios with validated computer codes demands more experimental information on the basic properties of the interactions between the materials involved. To achieve this we investigated the heat transfer between a wire spaced fuel-bundle and the coolant on the one hand in order to find the coolability limits and the chemical behaviour of a mixture of fuel, cladding material and the HLM coolant on the other hand. The fuel-coolant compatibility experiments were done using $\mathrm{UO}_{2}, \mathrm{PuO}_{2}$ and unirradiated MOX fuel. The energy release, the solubility of the materials in the coolant and the formation of compounds with the fuel, coolant and cladding materials was investigated. Furthermore, the dispersion of fuel particles in the coolant, which is a possibility in case of complete clad failure, was simulated using an appropriate numerical approach. Here the goal was to address the migration of the fuel and potential criticality problems due to fuel accumulation. As a final objective of the SEARCH project, the prevention of risk to the general public was studies by investigating the potential release of radioactive elements, including heavy volatile activation and spallation products such as Po and
$\mathrm{Hg}$, and fission products into the environment. For this purpose the kinetics and efficiency of procedures to capture these elements in the cover gas was examined. In addition the evaporation rate of $\mathrm{Hg}$ and $\mathrm{Po}$ from the Lead-bismuth eutectic coolant was measured in order to obtain a full experimental data-set for licensing. Issues related to Po management were addressed as well using an ab initio theoretical approach. Using this method it was possible to predict the Po solubility in LBE, to address the interaction with noble metals helping to select possible getters and to study the formation of Po compounds.

\subsection{FP7 SEARCH - main achievements}

The main achievements of SEARCH project have been the following. Firstly, heat transfer test of a wire-spaced fuel bundle mock up in forced and natural convection were performed. Here, a heat transfer correlation was established that can be used for further analyses of the reactor design. Secondly, significant steps were taken in the development of impurity and oxygen control techniques and methods were taken. The impurity source terms from corrosion and spallation were determined and mechanical and cold trap filtering tests were performed. The project also showed the compatibility of homogenous and sintered MOX fuel with LBE at 500 and $800^{\circ} \mathrm{C}$. In these tests the pellet integrity was maintained completely and no compound from chemical interactions between the lead bismuth coolant and the MOX fuel were found.

The project also built CFD and Simmer models for fuel dispersion studies where particle transport studies, accumulation zones were determined. Finally, the project measured the release of $\mathrm{Hg}$ and $\mathrm{Po}$ from LBE where in the case of $\mathrm{Hg}$ it studied the ideal behaviour while in the case of Po it studied the dependence on the covergas and LBE oxygen content. We found that volatile molecules are formed with water vapour but also that the Po compounds for a stable deposition on steel below $300^{\circ} \mathrm{C}$.

During the project, two workshops and one lecture series were organized.

\subsection{MAX (MYRRHA Accelerator eXperiment)}

The present FP7 proposal MAX [16] is subsequent to the recommendations of the Strategic Research Agenda of SNETP for ADS development in Europe. It is aimed to pursue the R\&D required for a high-power proton accelerator as specified by the MYRRHA project. There is especially a strong focus on all the aspects that pertain to the reliability and availability of this accelerator.

This R\&D effort builds on the large body of results and the clear conclusions that have been obtained during the consecutive FP5 project PDS-XADS and FP6 project EUROTRANS.

\subsection{FP7 MAX - main achievements}

With respect to the EUROTRANS outputs, a very significant progress has been made on the path towards the accelerator for MYRRHA. From the very start, MAX has been organized around the actual needs of the 
MYRRHA Linac and thereby has been able to focus on the specific requirements of this machine. This has led to a number of achievements that are all fundamental in view of the reliability goal:

- a fully reliability oriented overall consolidated concept of the accelerator;

- a set of benchmarked modelling tools allowing for startto-end beam simulations;

- an operational reliability model based on the SNS experience;

- an adequate and realistic injector design;

- a detailed engineering design of a few critical elements.

Specific experiments, matched to particular aspects of an ADS-accelerator, have also supported some of these achievements or provide valuable information for future and further developments:

- cooling performance tests of the 4-rod RFQ model cavity in real CW RF operation;

- investigation of the behaviour of a low- $\beta$ elliptical superconducting (SC) cavity in accelerator-like conditions ( $2 \mathrm{~K}$, high $\mathrm{RF}$ power);

- assessment of a SC cavity fault-recovery scenario using a digital low level RF feedback system and featuring an adaptive tuner controller;

- RF test of a superconducting $\mathrm{CH}$ cavity at $4 \mathrm{~K}$ and $2 \mathrm{~K}$ in vertical cryostat;

- performance of a $704 \mathrm{MHz}$ solid state RF amplifier module and associated power combiner.

A particularly strong achievement of the results generated by the MAX programme is the global level of confidence, in the concept on the one hand, and in the feasibility of its components on the other hand. This level of confidence is coherent with the fact that MAX has now brought to the first major milestone on the road towards the realisation of the MYRRHA Linac, this milestone being labelled "ready for prototyping". It is the starting point of a new set of mandatory R\&D activities where the emphasis should lie on experimental optimisation.

\subsection{FREYA (Fast Reactor experiments for hYbrid Applications)}

The objective of the FREYA project was twofold. One part of the project focussed on the validation of the methodology of sub-criticality monitoring that is envisaged for the online sub-criticality monitoring in ADS systems like MYRRHA. The other part of FREYA was dedicated to validate the computer codes and nuclear data that are used for LFR critical cores like ALFRED and MYRRHA. In the first stage of the project, the sub-criticality measurement method that were investigated in the MUSE FP5 experiments in the MASURCA facility had to be tested in several simple VENUS-F lead cores with different sub-criticality levels and coupled with the GENEPI-3C deuterium accelerator. Next, the selected sub-criticality methods were investigated in more complex VENUS-F cores simulating the MYRRHA core in more detail. This included robustness tests for variations in source position and reflector material. Finally, a special "island" to simulate the LFR ALFRED core in VENUS-F was assembled to validate the computational tools in critical experiments.

\subsection{FP7 FREYA - main achievements}

The main achievements of FREYA project can be summarized as follows.

Several VENUS-F fast reactor cores were coupled to a GENEPI-3C accelerator that delivers a deuteron beam. GENEPI-3C provides an external neutron source to the VENUS-F reactor through $\mathrm{T}(\mathrm{d}, \mathrm{n}) 4 \mathrm{He}$ fusion reactions. Different sub-criticality levels of the VENUS-F fast core for the nominal operation mode of ADS (k-eff varied 0.950.99 ) as well as a deeper subcritical level of 0.90 (core loading) were studied. The applicability of the different sub-criticality measurement techniques was investigated. FREYA experimental programme with regard to the LFR as well as for the critical mode operation of MYRRHA for the licensing of these designs so as for the validation of reactor codes has been accomplished. Six workshops were held as well as a one week dissemination lab-session.

\subsection{ARCAS (ADS and fast Reactor CompArison Study)}

The objective of the proposal is based on the outcome of PATEROS CA to assess more in depth the regional approach to $\mathrm{P} \& \mathrm{~T}$ implementation. It will respond to one of the key-topics put forward by the Strategic Research Agenda of SNETP. The project intends to look at the economical aspects of the most realistic scenario for $\mathrm{P} \& \mathrm{~T}$ with the hypothesis: limit the MA bearing fuel transport and limit the MA bearing fuel handling in and between all places such as at the reactor, at the fuel fabrication and at the reprocessing plant. We would like to assess the cost associated to implementing ADS's or dedicated Fast Reactors as minor actinide burning facilities. The idea is to start from two fixed hypotheses: (1) we work in doublestrata approach and look only at the second ("burning" stratum); (2) we assume a certain influx of minor actinide mass per year that needs to be burned. These two hypotheses will allow the project to avoid extensive scenario studies.

The economic impact will be evaluated for investment cost, associated fuel cycle and operational cost but not the needed R\&D cost. A crucial parameter to be established for both reactor systems is the maximal minor actinide (MA) content in a core loading. This maximal MA value is determined by operational safety criteria to be adhered by the dedicated burner. An evaluation of a number of safety parameters for the systems will give an upper boundary for the minor actinide mass present in the core.

In order to not diversify the work, the project should define a generic and representative system for the ADS approach and the FR approach. For the ADS, one can benefit from the work done in the FP6-EUROTRANS on the EFIT design. For the FR, one could use an SFR or LFR as a starting point. However, the design should be optimized to the task of a dedicated burner. Concerning the FR two options could be envisaged for the core lay-out: driver fuel with blanket or homogeneous mixture. 


\subsection{FP6 ARCAS - main achievements}

ARCAS project main achievements have been: establishing a reference minor actinide stream for a European region eligible for transmutation; study of homogeneous and heterogeneous transmutation in sodium-cooled Fast Reactor from FP7-CP-ESFR; study of homogeneous transmutation in lead-cooled Accelerator Driven System EFIT from FP6-IP-EUROTRANS; state-of-the-art report on transmutation fuel fabrication and reprocessing, including Technological Readiness Levels; scenario studies, including economic assessment, of transmutation in a regional European frame work.

\section{FP7 conclusions}

In this paper we tried to summarize the importance of the EURATOM Framework Programme acting as a trigger to foster the national efforts together with the DG RTD framework programme for reaching serious progress in a demanding $R \& D$ programme in terms of diversity of needed skills and competencies, various experimental unique facilities and laboratories as well as in financial means needed for such an endeavour aiming to industrializing a full concept of closing the fuel cycle in a European regional approach with different national policies towards nuclear energy.

The enormous work sitting behind these projects would not have been possible without the long standing support including financial one from the EURATOM DG RTD framework programmes since the FP5 and continued in FP6, FP7 and H2020 for which the authors on behalf of the community they represent are very thankful.

\section{References}

1. OECD/NEA, Accelerator-driven systems (ADS) and fast reactors (FR) in advanced nuclear fuel cycles: a comparative study, OECD/NEA, 2002
2. OECD/NEA, Potential benefits and impacts of Advanced Nuclear Fuel Cycles with actinide partitioning and transmutation, OECD/NEA, 2011

3. OECD/NEA, Impact of advanced Nuclear fuel cycle options on waste management policies, 2006

4. OECD/NEA, Advanced Nuclear Fuel cycles and radioactive waste management, OECD/NEA, 2006

5. H. Oigawa, T. Yokoo, K. Nishihara, Y. Morita, T. Ikeda, N. Takai, Parametric survey for benefit of partitioning and transmutation technology in terms of high-level radioactive waste disposal, J. Nucl. Sci. Technol. 44, 398 (2007)

6. H. Aït Abderrahim, ADOPT final report - Recommendations for the EC for further activities in P\&T and ADS development. ADOPT Thematic Network (FIKW-CT-200120178), 2005

7. M. Salvatores, V. Meyer, V. Romanello, A. Boucher, A. Schwenk-Ferrero, PATEROS D2.2: Results of the scenario studies, 2008

8. F. Venneri, C. Bowman, R.A. Jameson, Accelerator-driven transmutation of waste (ATW), a new method for reducing the long-term radioactivity of commercial nuclear waste, 1993

9. CEA, Bilan des recherches conduites sur la séparationtransmutation des éléments radioactifs à vie longue et sur le développement de réacteurs nucléaires de nouvelle génération. CEA, 2012

10. Experimental Facilities in Support of Liquid Metal Cooled Fast Neutron Systems. A Compendium, available at https:// nucleus.iaea.org/sites/lmfns/Pages/default.aspx (accessed September 13, 2019)

11. OECD/NEA, State-of-the-art report on the progress of nuclear fuel cycle chemistry, OECD/NEA, 2018

12. OECD/NEA, Review of operating and forthcoming experimental facilities opened to international $\mathrm{R} \& \mathrm{D}$ co-operation in the field of advanced fuel cycles, OECD/NEA, 2019

13. Nuclear Innovation 2050 (NI2050), available at https:// www.oecd-nea.org/ndd/ni2050/ (accessed September 13, 2019)

14. https://maxsima.sckcen.be/

15. https://cordis.europa.eu/project/id/323312

16. J-L. Biarrotte et al., MAX FP7 final report summary, https://cordis.europa.eu/project/id/269565

Cite this article as: Hamid Aït Abderrahim, Peter Baeten, Alain Sneyers, Marc Schyns, Paul Schuurmans, Anatoly Kochetkov, Gert Van den Eynde, Jean-Luc Biarrotte, Partitioning and transmutation contribution of MYRRHA to an EU strategy for HLW management and main achievements of MYRRHA related FP7 and H2020 projects: MYRTE, MARISA, MAXSIMA, SEARCH, MAX, FREYA, ARCAS, EPJ Nuclear Sci. Technol. 6, 33 (2020) 